\title{
Request for a Test Exposure of OPERA Targets in the NuMI Beam
}

\author{
K. Kodama \\ Aichi University of Education, Kariya, JAPAN \\ G. Tzanakos
}

University of Athens, Athens 15771, GREECE

B. Baller, B. Lundberg, R. Rameika, N. Saoulidou

Fermilab, Batavia, IL 60510, USA

S. Aoki

Kobe University, Kobe, JAPAN

D. Autiero, L. Chaussard, Y Caffari, Y. Déclais,

I. Laktineh, J. Marteau, P. Royole-Degieux

IPNL, IN2P3-CNRS and Université C. Bernard Lyon I, Villeurbanne, FRANCE

K. Heller

University of Minnesota, Minnesota, MN, USA

K. Hoshino, M. Komatsu, M. Nakamura, T. Nakano,

K. Narita, K. Niwa, N. Nonaka, O. Sato, T. Toshito

Nagoya University, Nagoya, JAPAN

and

D. Naples, V. Paolone

University of Pittsburgh, PA 15260, USA

November 2004 


\begin{abstract}
We request to use the Fermilab NuMI neutrino beam in the MINOS Near Detector Hall to produce neutrino interactions in two separate detector arrangements using prototype target bricks designed for the OPERA experiment. OPERA is scheduled to begin taking data in the CERN Neutrinos to Gran Sasso (CNGS) beam in 2006.

The proposed test set up would be located just upstream of the MINOS Near Detector. The data will be used to validate the OPERA analysis scheme and to study backward particle production in neutrino interactions, which is of interest to the OPERA collaboration as well as the neutrino community in general. In addition, we contend that the data taken in this exposure may also be useful to the MINOS collaboration as additional input to the understanding of the initial composition of the neutrino beam. Ideally, this exposure could begin in early to mid-2005 and remain in place throughout 2005 . We anticipate providing timely feedback to both the OPERA and MINOS collaborations.
\end{abstract}

\title{
1 Introduction
}

The advantages of using nuclear emulsion as a particle detector are well known. The high resolution of emulsion has made it a medium of choice for a number of applications where the required spatial and angular resolution are paramount and its limitations due to the lack of timing information are less important. Emulsions are commonly used in cosmic ray detectors and have found applications in high energy experiments for detecting short lived particles such as charm, beauty and tau. The addition of electronic detectors to emulsion experiments solved the problem of the lack of timing information in the emulsion, but it was the development of automatic scanning machines that revolutionized the use of these hybrid detectors, making them capable of performing even in high rate environments. Most recently, the DONUT experiment (FNAL E-872), used a hybrid emulsion spectrometer to make the first direct observation of tau neutrino interactions [1].

The CNGS facility is being constructed to deliver a $\nu_{\mu}$ beam from the CERN SPS to the Gran Sasso Laboratory. Since it is believed that $\nu_{\mu} \leftrightarrow \nu_{\tau}$ oscillations explain the observed atmospheric $\nu_{\mu}$ deficit, the CNGS beam, coupled to a detector capable of observing $\tau$ appearance is an important 
experiment in the context of the world wide effort to determine the neutrino mass mixing matrix. The OPERA detector has been optimized to detect a significant sample of $\nu_{\tau}$ interactions by the subsequent observation of $\tau$ production and decay [2].

The OPERA target is a massive emulsion detector made in a sandwich structure of lead plates and layers of nuclear emulsion. For historical reasons this arrangement has been called an Emulsion Cloud Chamber or ECC. The ECC concept, which has many advantages over the use of bulk emulsion, has been used in the DONUT experiment. The ECC detector is capable of measuring non-nuclear fragment individual tracks, coming from the primary neutrino interaction vertex with their three dimensional slopes and momenta. It is also capable of electron identification with good $\mathrm{e} / \gamma$ separation, due to its very fine segmentation. The OPERA ECC target modules are constructed as bricks of dimensions $12.5 \times 10.0 \times 7.5 \mathrm{~cm}^{3}$ in horizontal, vertical and along the beam axis. Each brick consists of series of a $56(1 \mathrm{~mm}$ thick) plates of passive material (lead or iron) alternated with emulsion films ( $43 \mu \mathrm{m}$ emulsion layer on both sides of a transparent $200 \mu \mathrm{m}$ thick plastic sheet). See Figure 1.

In preparation for OPERA we would like to expose the OPERA target modules to a beam of neutrinos. This will allow us to test many. of our analysis procedures and techniques as well as to validate the simulation of neutrino interactions, both for the production of forward and backward particles. Although the ME (medium energy) beam of NuMI would be a better match to the CNGS energy, data acquired with the NuMI LE (low energy) beam would serve the same purpose, albeit more challenging. Given the high interaction rate from the NuMI beam, the test detector target mass can be kept low and additional detectors can easily be built around a small target. These type of measurements are not possible in the CNGS beam, since it has no short baseline hall.

\section{The Detectors}

For the requested exposure two independent target setups are proposed. They would be arranged side-by-side at the same $z$ location. The first one, labeled EDDS, uses multiple ECC bricks and the DONUT scintillating fiber tracker (SFT). This configuration is optimized for the detection of a large number of events. The second set up, labeled SBHD, uses a single ECC brick 
which will be able to measure a few events but in a very detailed way. The positioning of the setups with respect to the MINOS Near Detector is shown in Figures 2 and 3.

For both detectors, EDDS and SBHD, muon identification will be performed by the electronic tracking detectors whose data is linked by time stamps to events recorded in the MINOS Near Detector. We do not request direct access to the MINOS data, but rather only need the muon and calorimetric information for a list of spills for which we will provide the time stamp.

The following two sections describe each of these configurations in more detail.

\subsection{ECC-DONUT Detector Setup (EDDS)}

For this set up we will use 64 bricks segmented into four walls of four (horizontal) by four (vertical) bricks. The downstream surface of each wall will be covered with a thin "changeable" emulsion sheet (CS) and 2 orthogonal $(x-y)$ planes of scintillating fibers. The CS is an $800 \mu \mathrm{m}$ plastic sheet with a $100 \mu \mathrm{m}$ emulsion film on each side. A CS is mounted $1 \mathrm{~cm}$ downstream of each ECC brick wall, and is replaced periodically when the track density exceeds a predetermined level.

The fiber planes are the same ones used in the DONuT detector [3]. Each plane is $0.56 \mathrm{~m} \times 0.56 \mathrm{~m}$, and the fibers are $500 \mu \mathrm{m}$ diameter made from SCSF78 produced by Kuraray Co. Figure 4 shows a schematic of the brick wall and SFT arrangement.

The SFT planes are readout by image intensifiers and CCD cameras. Since the SFT readout by the Image Intensifier chain is too slow to separate events in a spill, the data from the CCD camera is read out at the end of the beam spill by a local PC computer and stored on disk. A network connection to the PC will be used to control the PC and to transfer data off of the disk.

For most of this exposure we plan to use lead as the passive material in the bricks in order to achieve the best performance for the momentum measurement obtained through multiple-scattering and the electron ID. However since the MINOS detector target material is iron, to check the number of shower tracks and emission angles for neutrino-iron interaction, we also plan to expose iron ECC bricks. With iron, the momentum measurement accuracy and electron ID efficiency will decrease due to the 3 times longer radiation 
length compared to lead.

This setup has already been constructed at Nagoya University in Japan. This brick arangement is called a Mini-Wall structure, specifically designed to be similar to the installation in OPERA. Figure 5 is a photo of the setup in Nagoya. The setup as shown in the photo will be shipped from Nagoya. Both the SFT planes and the Mini-Walls are supported by a frame of overall dimensions $1.8 \mathrm{~m}$ high, $1.8 \mathrm{~m}$ wide and $1.2 \mathrm{~m}$ along the beam axis. The target mass of the 64 ECC bricks is $531.2 \mathrm{~kg}$. The mass of the aluminum frame is less than $300 \mathrm{~kg}$. Some additional structural support will be needed to raise the setup to beam height.

\subsection{Single Brick Hybrid Detector (SBHD)}

In this set up we will construct a small hybrid detector around a single ECC brick. This will allow us to study in fine detail a set of a few hundred neutrino interactions by combining the measurements performed in the ECC brick with the ones performed in the tracking and calorimetric detectors surrounding the brick. The purpose of this exposure will be mainly for cross-checking the OPERA simulation and analysis procedures, but many of the results obtained could be of general interest in the neutrino community since they are affected by nuclear effects in neutrino interactions, particularly important in the case of lead nuclei.

The hybrid setup (see Figure 6) includes one ECC brick, precise tracking in the forward and backward directions, backward detection of neutrons and photons, and measurement of forward electromagnetic energy. All of the detectors in this set up are made of recycled equipment from the NOMAD, CMS and OPERA experiments. These will be shipped from Europe.

The dimensions of each component are quite small, given the fact that they have to match the acceptance of the particles coming from just one ECC brick. The footprint of the entire setup will cover an area of $1.5 \mathrm{~m}$ along the beam direction and $1 \mathrm{~m}$ transverse to the beam. The detectors will be mounted on a light mechanical structure with an upper level which will support the detector centered at the beam height, while the electronics will be placed on the structure below the detector.

The central part of the SBHD is the single ECC brick which will be placed in between two silicon trackers. Each tracker provides 3 views in $x$ and 3 in $y$. The silicon trackers will measure precisely the directions of all the 
charged particles exiting the brick in the forward and backward directions. This telescope, with an active surface equivalent to the one of a brick, is build out of 12 single sided silicon sensors readout with strips with a pitch of $183 \mu \mathrm{m}$. The silicon detectors are readout through an optoelectronic chain down to a card hosted in a VME $9 \mathrm{U}$ bin. Further description of the readout process is found in [5] The VME bin is readout by a PC via a VME to PCI interface.

Downstream of the forward silicon tracker will be a few towers (each with a cross section of about $10 \times 10 \mathrm{~cm}^{2}$ and a length of $50 \mathrm{~cm}$ ) of a lead glass electromagnetic calorimeter. These towers will be readout with an ADC module inserted in the VME bin.

Upstream of the backward silicon tracker will be a backward neutral detector (BND). The BND will be built with short pieces of the scintillator strips used for the construction of the OPERA target tracker. These strips have a thickness of $1 \mathrm{~cm}$ and a width of $2.6 \mathrm{~cm}$. The cross section of the detector will be $20.8 \times 20.8 \mathrm{~cm}^{2}$ and the length about $20 \mathrm{~cm}$. It will consist of 48 planes of $1 \mathrm{~cm}$ thickness, perpendicular to the beam direction. Each plane will be made out of 8 strips. The planes will have their strips alternatively aligned along the $x$ or the $y$ view. The strips will be read out with wave length shifting fibres, from one side only. The 384 fibers coming out of the detector will be read out by 6 Hamamatsu M64 photomultipliers coupled to the standard OPERA target tracker electronics. This is composed by a system distributing a time stamp to 6 front-end cards (one per photomultiplier). The front end cards output the data to a local ethernet network made by a switch connected to a PC. The event reconstruction is performed on the PC on the basis of the time stamp of the events.

Upstream of the first two planes of the BND we will put a thin layer of lead to be used as a preshower for the backward photons. The neutrons will be detected in the bulk of the scintillators planes after the lead foil. The BND detector will have an efficiency of about $60 \%$ for the detection of backward neutrons. Finally in the most upstream position will be a scintillator veto plane.

The trigger logic will be based on the presence of a signal in the electromagnetic calorimeter in absence of signal in the veto. This trigger will be setup with NIM modules and distributed to the readout of the silicon telescope, the front end cards of the PMs for the BND readout and to the $\mathrm{ADC}$ for the ECAL readout. We plan to use two $\mathrm{PCs}$, one for the readout 
of the BND and one for the readout of the silicon telescope and the ECAL trough the VME bus. The global event building will be performed with an ethernet based DAQ. The needed electronics will be included in one VME $9 \mathrm{U}$ bin and one NIM bin. The timing module will be located in the VME bin and it can accept as input standard PPS and IRIG-B signals from a GPS system. These signals will have to be provided by the local GPS system used for the MINOS Near Detector. The data will be stored locally on the disks of the PCs.

During the $1.87 \mathrm{~s}$ of the spill cycle the offline analysis will be able to determine, on the basis of the pattern of tracks recorded by the silicon trackers, if the trigger was really related to a neutrino interaction which occurred in the brick or an interaction in the ECAL, in the backward neutrals detector or in some mechanical supports. The connection with the part of the event measured in the MINOS near detector will be performed on the basis of the GPS time stamp.

The forward silicon tracker will perform the role of a changeable sheet, which will allow us to reduce the scanning load by scanning the first emulsion sheet just in a small area around the prediction of the reconstructed track. The silicon tracker will also allow us to study the possible role of the changeable sheets in the confirmation of the event location in the brick.

The other interesting applications of the data taken with this setup will be related to the comparison between the kinematical reconstruction performed in the brick (thanks to the measurement of the momenta of charged particles trough their multiple scattering and the electron and photon reconstruction through the sampling of their showers) and the ones performed by the muon reconstruction and the calorimetric measurements outside the brick.

\section{The NuMI Beam}

The NuMI target hall was designed to accomodate variable target and focusing horn configurations such that the neutrino beam energy would be tunable. Current measurements of the $\nu_{\mu} \rightarrow \nu_{\tau}$ mass difference, $\Delta m_{23}^{2}$, that the MINOS experiment intends to measure, indicate that the low energy (LE) beam configuration for the target and horns will provide the maximum sensitivity to the measurement. The nominal LE configuration will produce the interacted neutrino spectrum shown in Figure 7. 
Reconfiguration of the NuMI target hall to produce a higher energy beam, though doable, is very time consuming and unlikely to occur in the early years of MINOS running. However, it has been determined that higher energy beam spectra are easily produced by moving the target upstream relative to the start of the first horn. A displacement of $100 \mathrm{~cm}$ will produce a pseudo-medium enrgy (pME) beam and a displacement of 200 to $150 \mathrm{~cm}$ will produce a pseudo-high energy ( $\mathrm{pHE}$ ) beam. The interacted neutrino spectra from these two configurations are shown in Figure 8 . In addition to having higher mean energies, these beams also increase the integrated event rates by factors of 2 to 4 with respect to the nominal LE beam. The MINOS Experiment is currently developing a run plan to incorporate running in these higher energy configurations during the first year of data taking.

\subsection{Event Rates}

Following an initial commissioning period, the nominal proton intensity on the NuMI target is expected to be $2.5 \times 10^{13}$ protons per spill. A spill has an $11 \mu \mathrm{sec}$ duration and a $1.87 \mathrm{~s}$ repetition rate. The neutrino interaction rate for the LE beam configuration at this per spill intensity is expected to be $10^{-4}$ events per brick per spill. In a typical day of $4 \times 10^{4}$ spills, a single brick will accumulate between 4 and 12 interactions per day, depending on the target position. The length of time for which a brick will be exposed will depend upon the MINOS run plan, though we would anticipate changing out the SBHD brick at least once per day.

\subsection{Characteristics of Neutrino Interactions}

Monte Carlo distributions from neutrino interactions produced by the NuMI LE beam configuration are shown in Figures 9-11. These are the "true" values of multiplicity, momentum and angles, independent of any detector acceptance or resolution. The Monte Carlo interactions are generated using an iron target nucleus. The dotted line is for all events (i.e. from the spectrum of Figure 7. The solid line is for selection of events greater than 5 $\mathrm{GeV}$. 


\section{Installation and Operation Requirements}

We request to install our setups in early 2005, after the NuMI beamline commissioning phase has been completed. The installation scheme is simple for both brick configurations. The two independent parts of the detector will arrive from Europe and Japan already pre-assembled and tested. We request $10 \times 5 \mathrm{~m}^{2}$ space in the MINOS Service Building for final assembly and testing. We would then request three crane trips down to the hall to bring in the EDDS, SBHD and an additional frame constructed to lift up our detectors to beam height. The other parts (ECC bricks and host PCs) and electronics are brougth down by elevator. The transportation and installation of all these components both using the crane and the elevator (a couple of trips) will last at most one day.

Electrical requirements are summarized in Table 1 . It is a total of $3.25 \mathrm{~kW}$. Power for the EDDS is $120 \mathrm{~V}$; and $240 \mathrm{~V}$ for the SBHD equipment.

Table 1: Required power supply

\begin{tabular}{lr}
\hline EDDS & $120 \mathrm{~V}$ \\
\hline Readout II & $0.1 \mathrm{~kW}$ \\
NIM bin & $0.4 \mathrm{~kW}$ \\
DAQ host PC & $0.3 \mathrm{~kW}$ \\
misc. & $0.2 \mathrm{~kW}$ \\
\hline Sub total for $120 \mathrm{~V}$ & $1.0 \mathrm{~kW}$ \\
\hline \hline SBHD & $240 \mathrm{~V}$ \\
\hline NIM bin & $0.3 \mathrm{~kW}$ \\
VME bin & $1.0 \mathrm{~kW}$ \\
PCs & $0.6 \mathrm{~kW}$ \\
Various & $0.6 \mathrm{~kW}$ \\
\hline Sub total for $240 \mathrm{~V}$ & $2.5 \mathrm{~kW}$ \\
\hline Total & $3.25 \mathrm{~kW}$ \\
\hline
\end{tabular}

We need access to a public network connection. It is not used for DAQ itself but surely needed for the control of the DAQ PCs during our data taking. Data will be stored on the DAQ PCs disk. One $100 \mathrm{Mbps}$ (even 
$10 \mathrm{Mbps}$ ) port will be sufficient for our purposes for both detectors. For this exposure the event rate is much higher than in OPERA where we will remove bricks only after the electronic detector trigger has indicated that an interaction has occurred.

As soon as an emulsion brick is removed from the beam, the emulsion sheets are removed from the stack and developed. For this job we will need emulsion development space equivalent to the space in the New Muon Lab used for the DONUT experiment. Emulsion films will be processed using both developer( Fuji PDT-10) and fixer. Diluted acetic acid will also be used. In the MSDS and AIS of Fuji Photo Film Co. these typical developing solutins are not classified as dengerous materials. We estimate a need of about 100 liters of both developer and acetic acid, and about 400 liters of fixer. The solutions are collected as waste articles.

(The stacks may then be reconfigured with new films and returned to the test stand.) The developed films are then sent back to Nagoya University to await analysis.

\section{$5 \quad$ Emulsion Scanning and Event Analysis}

For the case of the SBHD brick we will take advantage of our high speed scanning system to fully scan each of the emulsion sheets. For the case of the bricks in the EDDS we plan to scan the whole surface of the CS. We will then use the reconstructed tracks in the CS to predict the location of interactions in the bricks. Event location in the bricks will then proceed using the same methods as in CHORUS and DONUT.

We have gathered a large amount of experience for the vertex analysis method, the so called NetScan, which was developed in the DONUT experiment and later applied to the CHORUS phase II analysis to more than 100,000 events.

NetScan is an analysis method similar to the ones used in standard electronic experiments. In each emulsion plate we reconstruct a track segment, determining its position and angle to sub-micon and milliradian precision respectively. The track segments are then reconstructed into tracks by offline programs to determine the complete event topology. structure. The event location efficiency is a function of the event multiplicity and hence neutrino energy. Traditionally only tracks with emission angle $\tan (\theta)<0.4$ are recon- 
structed. Increased scanning power allowing $0.4<\tan (\theta)<1.0$ will increase the event location efficiency as shown in Figure 13. For each reconstructed track the segment position and angular measurements are used to determine the track momentum from multiple scattering. Figure 12 shows the charged track momentum distribution from the DONUT interactions.

Electrons are identified by measuring the energy loss behavior and EMshower detection in the $10 X_{0}$ thick ECC brick[6]. Protons coming from the neutrino interaction vertex are identified by using $d E / d X$ information in the emulsion. Details of these event analyses are described in References [6] and [7].

\section{Conclusions}

We believe that the modest investment of effort described in this proposal will produce valuable measurements of the NuMI beam composition, while providing valuable data to the OPERA collaboration. The fine grained resolution of an emulsion detector allows one to actually see the neutrino interaction vertex and measure detailed properties of the interaction, something which is not possible in the course grained MINOS detector.

All the costs for this test exposure will be covered by ourselves, with no possible fallouts on Fermilab. We will request funds from the US-Japan agreement to cover preparation and installation costs incurred at Fermilab.

\section{References}

[1] K. Kodama et al., Physics Letters B, 504, 218-224, 2001

[2] Guler, M.et al.(OPERA Collaboration), CERN-SPSC-2000-028,2000.

[3] K. Kodama et al., Nucl. Instrum. Meth. A493, 45-66, 2002

[4] K. Kodama et al., Nucl. Instrum. Meth. A516, 21-33, 2004

[5] A. Lucotte, et al. ,Nucl. Instrum. Meth. A521, 378-392,2004

[6] K. Kodama et al., Rev. Sci. Instrum, 74, 53-56, 2003

[7] T. Toshito et al. Nucl. Instrum. Meth. A516,436-439,2004 


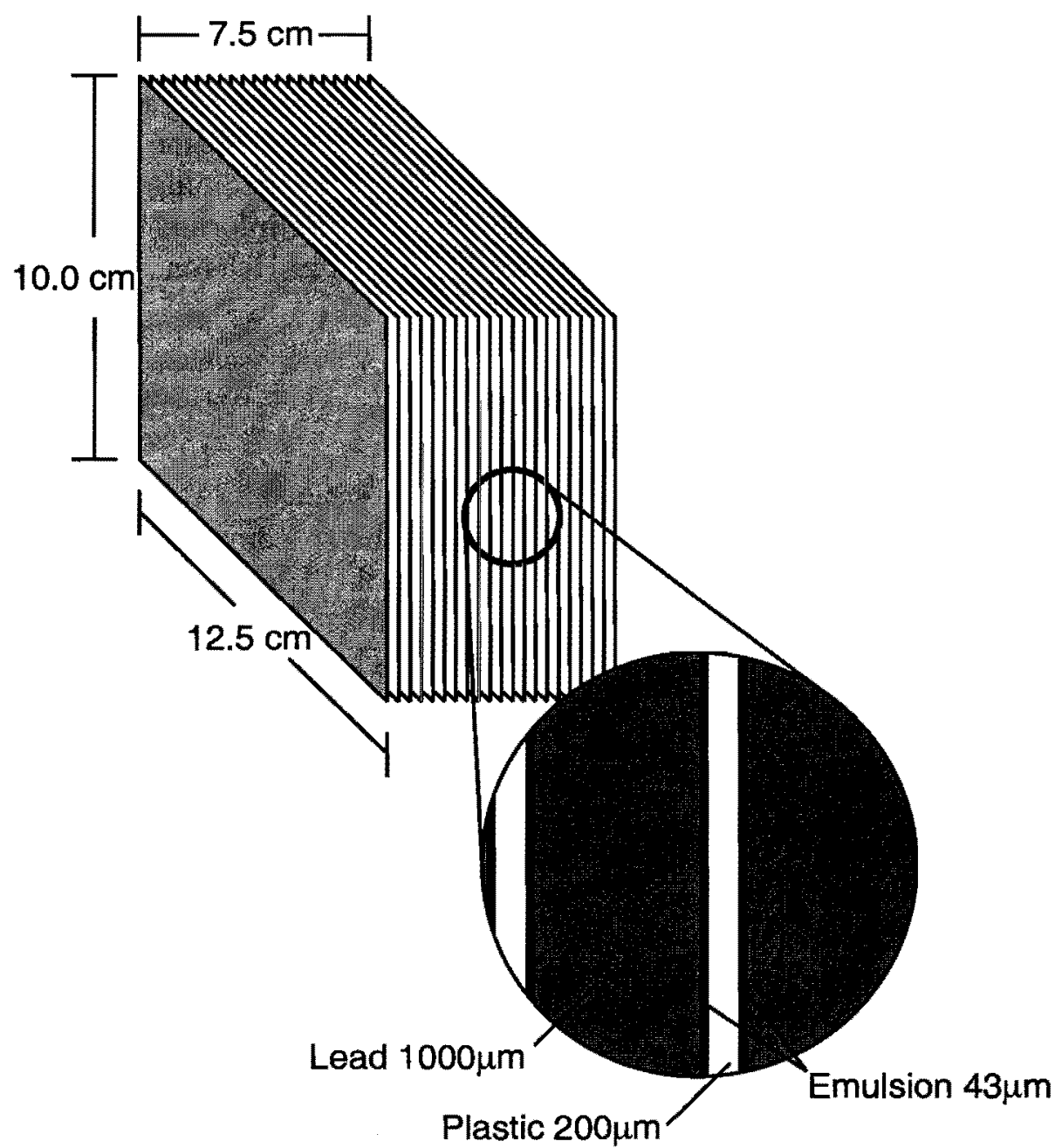

Figure 1: An OPERA ECC brick consists of $561 \mathrm{~mm}$ thick passive target material plates alternated with emulsion films ( $43 \mu m$ emulsion layer on both sides of a transparent $200 \mu m$ thick plastic film). The passive target material can be changed from lead to iron. 


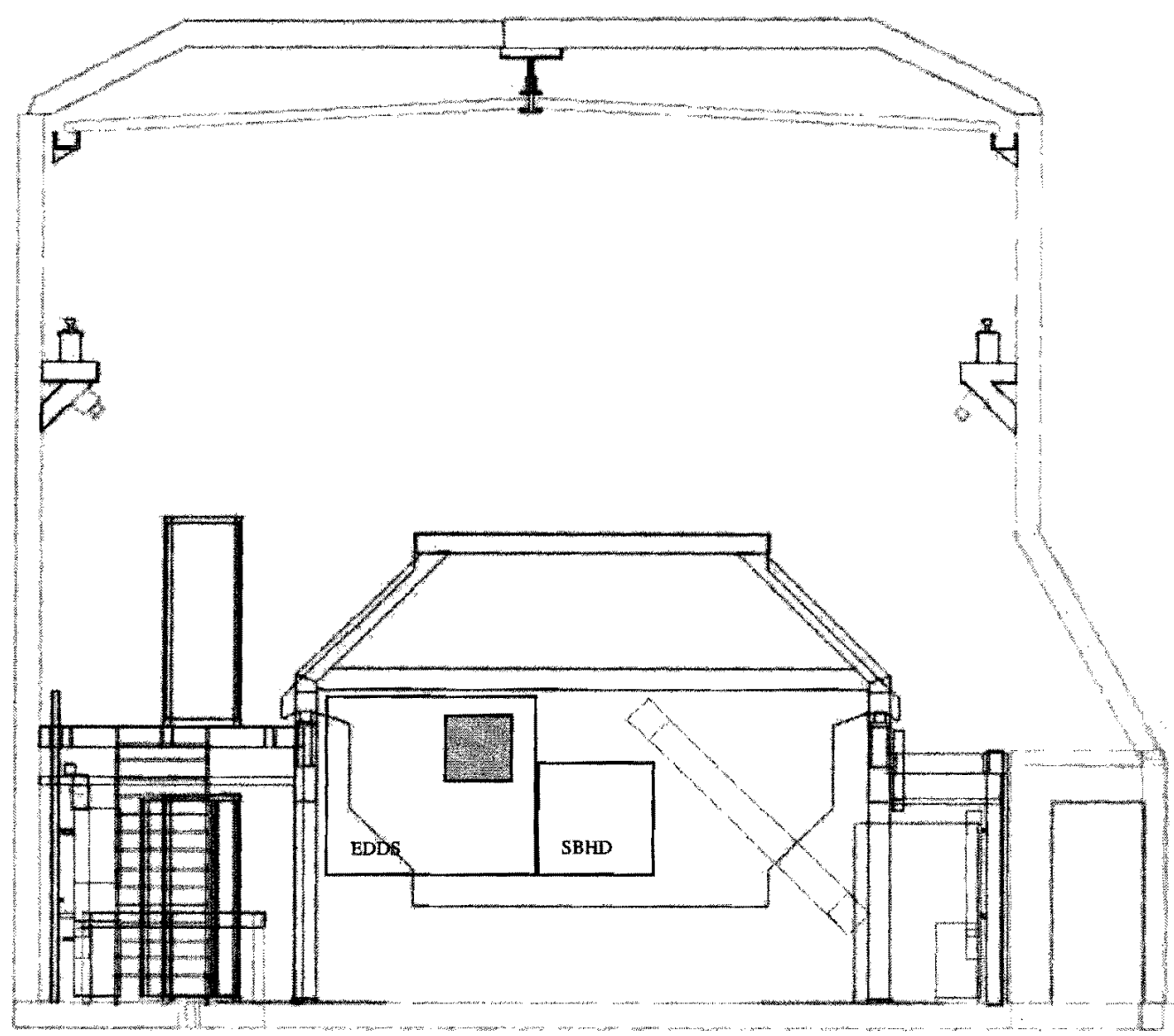

Figure 2: Location of detectors (EDDS and SBHD) with respect to the MINOS Near Detector. The beam is into the page in this cross section view. 


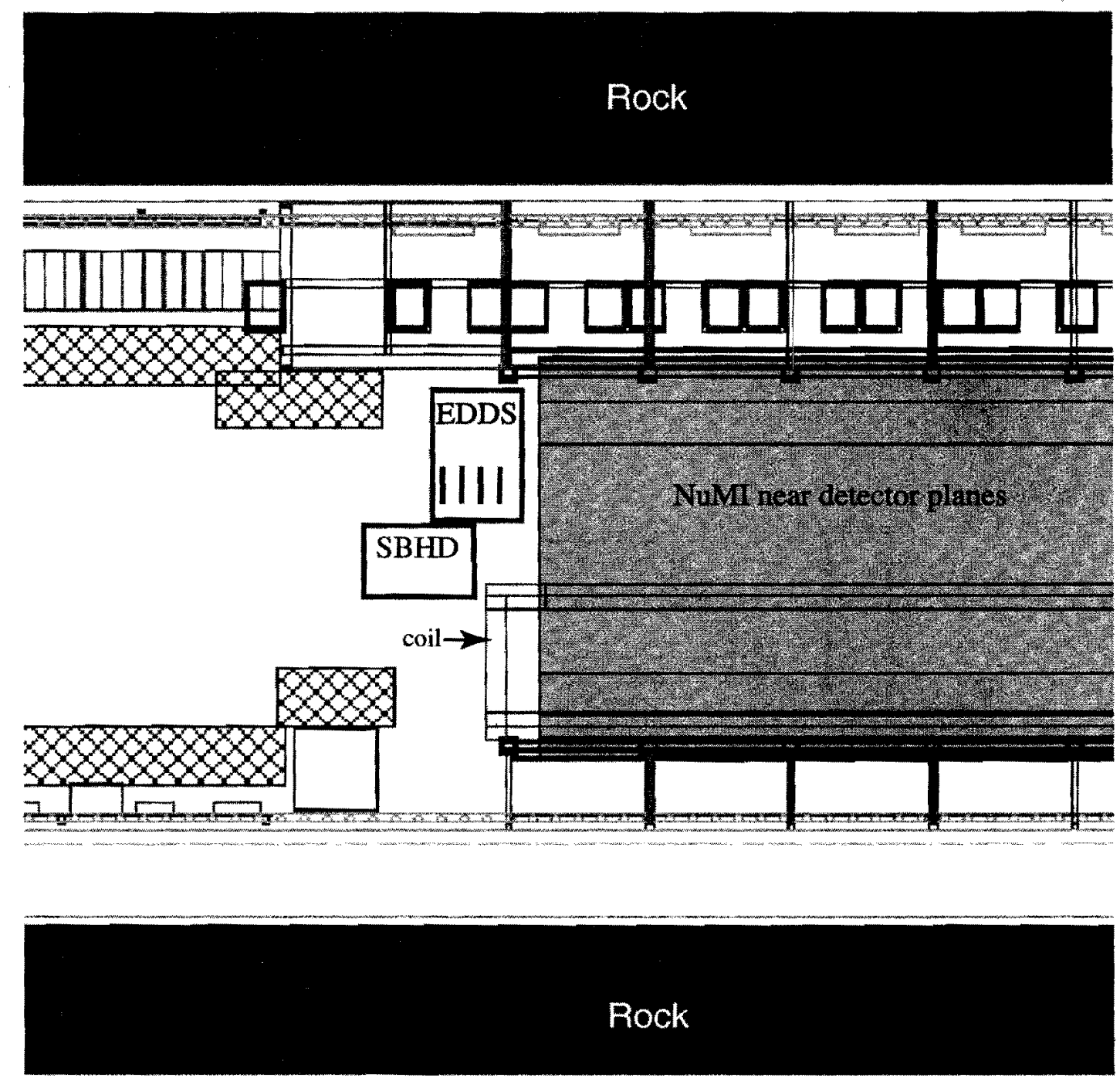

Figure 3: Location of test setups with respect to the MINOS Near Detector. Plan view 


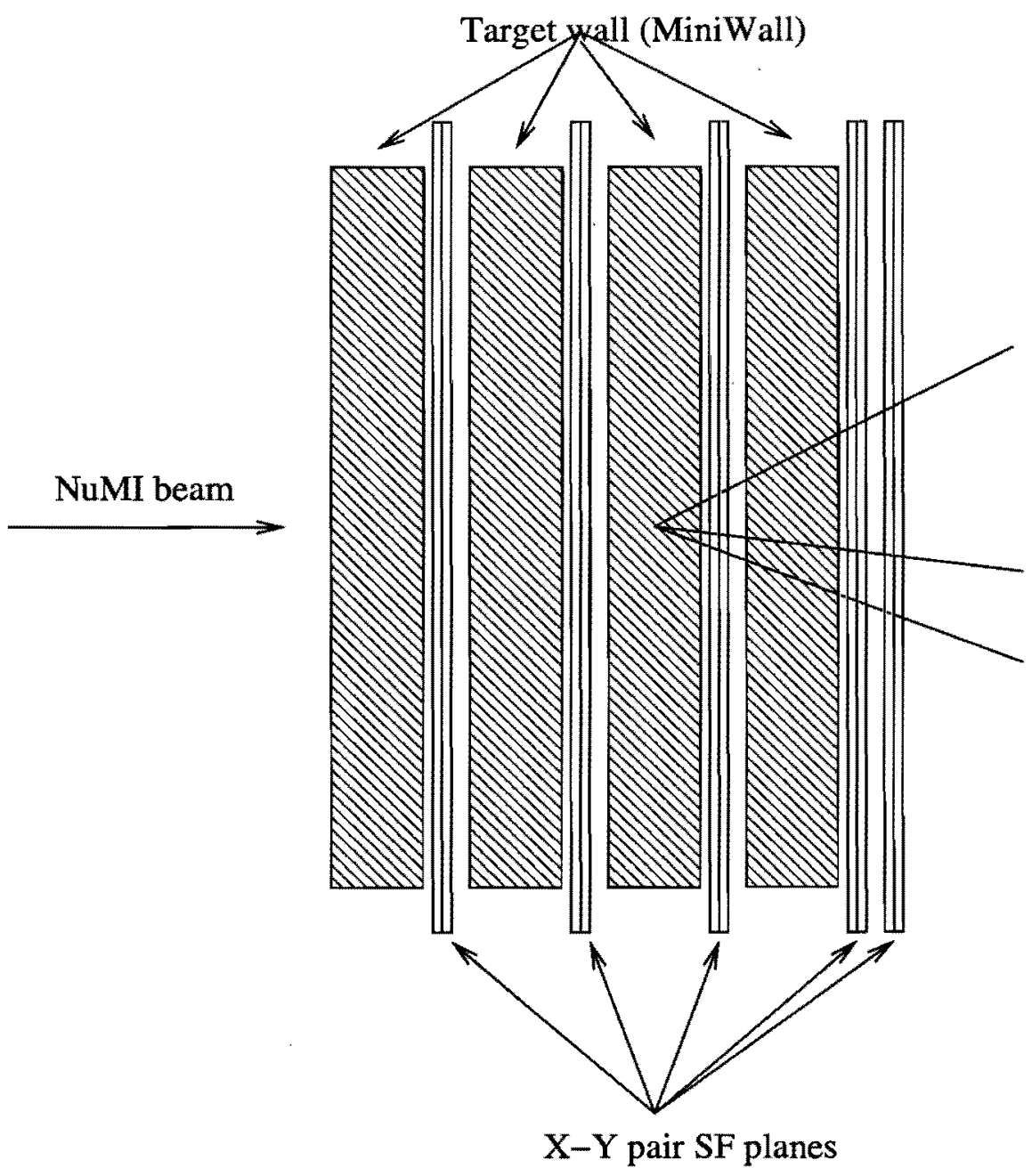

Figure 4: Schematic drawing of the EDDS Detector. 


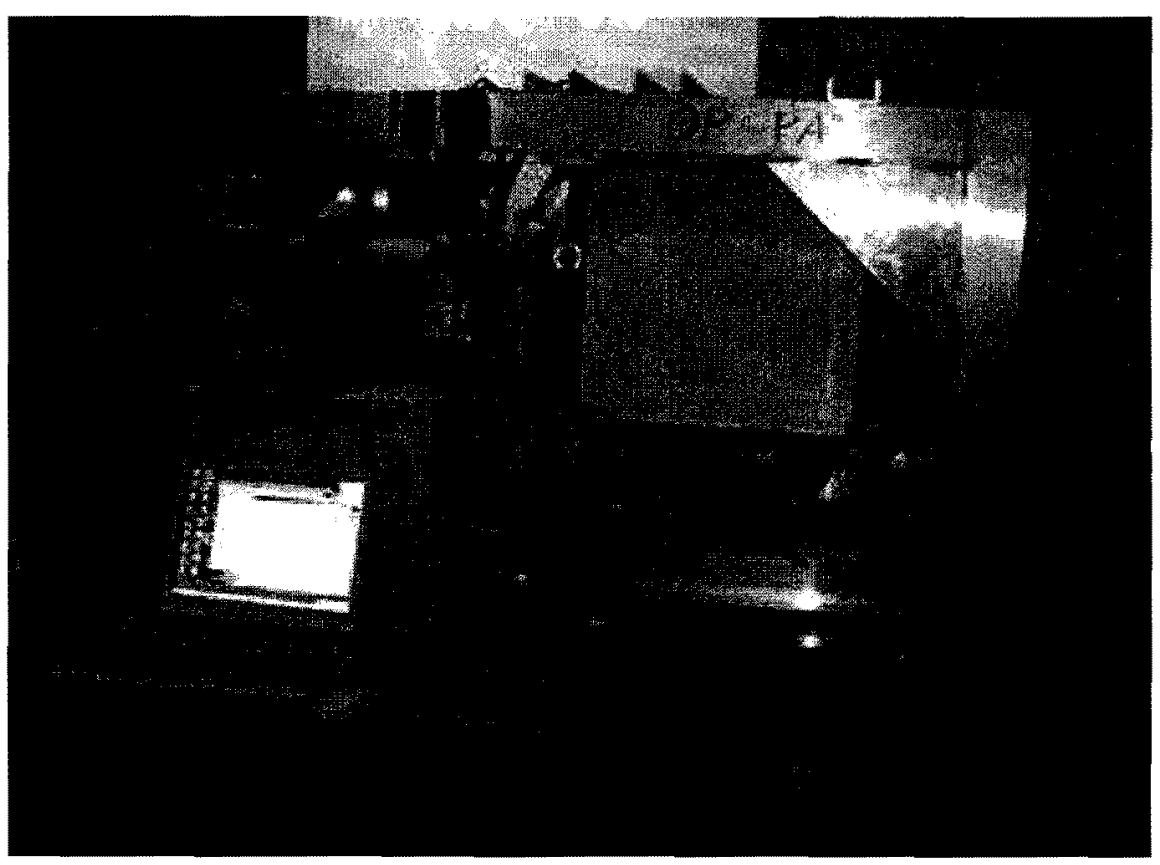

Figure 5: Picture of the EDDS taken at Nagoya University. 
Longitudinal dimensions: ECC * silicon trackers $20 \mathrm{~cm}$

Ecal (lead glass blocks) $50 \mathrm{~cm}$

Neutrea detector $50 \mathrm{~cm}$

Veto $5 \mathrm{~cm}$ (scinuillator planes)

Transverse dimensions: $\max 50 \mathrm{~cm}$, Si trackers will just exceed the brick by a few $\mathrm{cm}$ per side

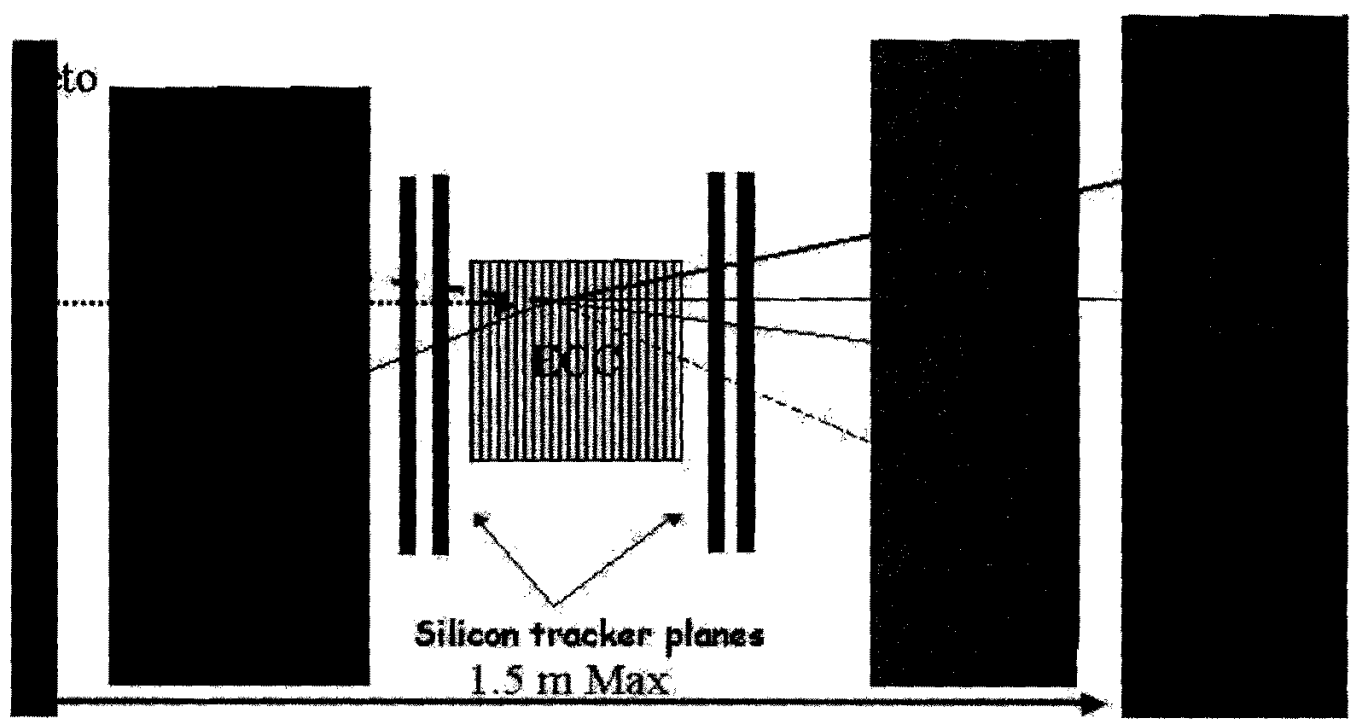

Figure 6: The Single Brick Hybrid Detector (SBHD). It includes one ECC brick, silicon tracking in the forward and backward directions, backward detection of neutrons and photons, forward electromagnetic calorimetry, forward hadronic calorimetry and muon identification from the MINOS near detector 


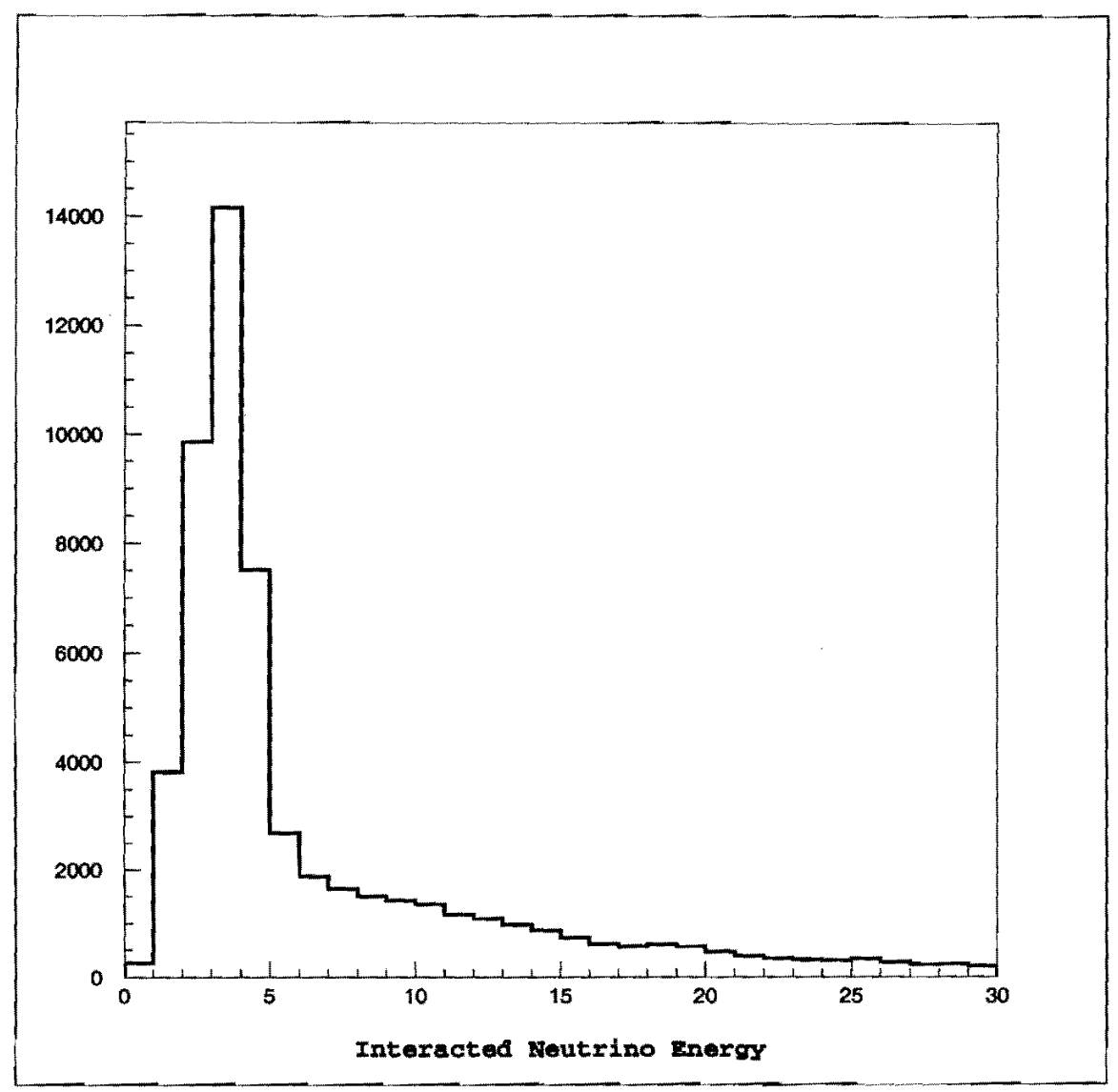

Figure 7: Interacted neutrino energy for $\nu_{\mu} \mathrm{CC}$ events with the NuMI low energy (LE) beam configuration 


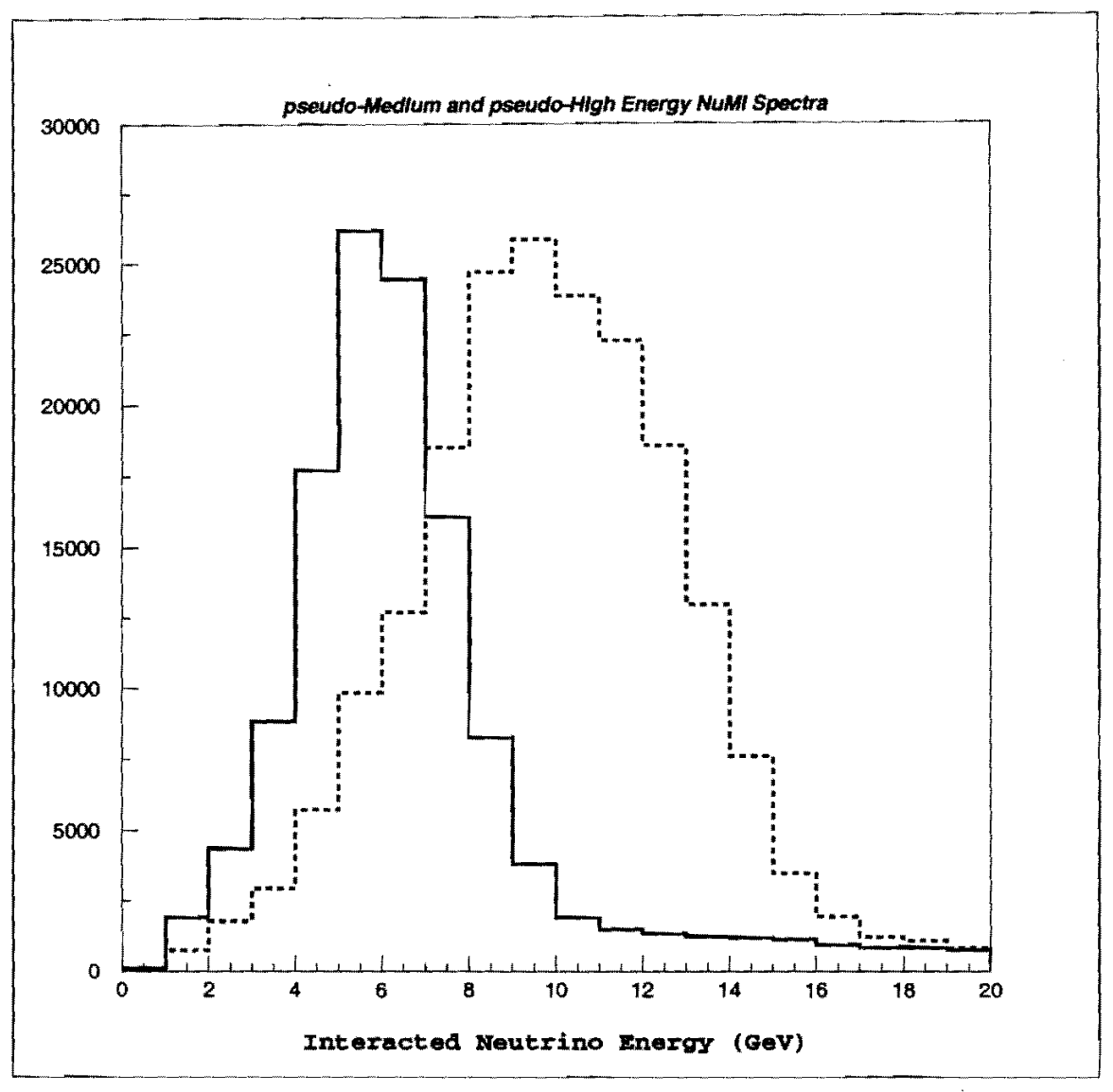

Figure 8: Interacted neutrino energy ( $\nu_{\mu} \mathrm{CC}$ events) for the NuMI pseudomedium and high energy configurations; the solid line is the spectra for the target displaced to $-100 \mathrm{~cm}$ and the dotted line corresponds to the target at $-250 \mathrm{~cm}$. 


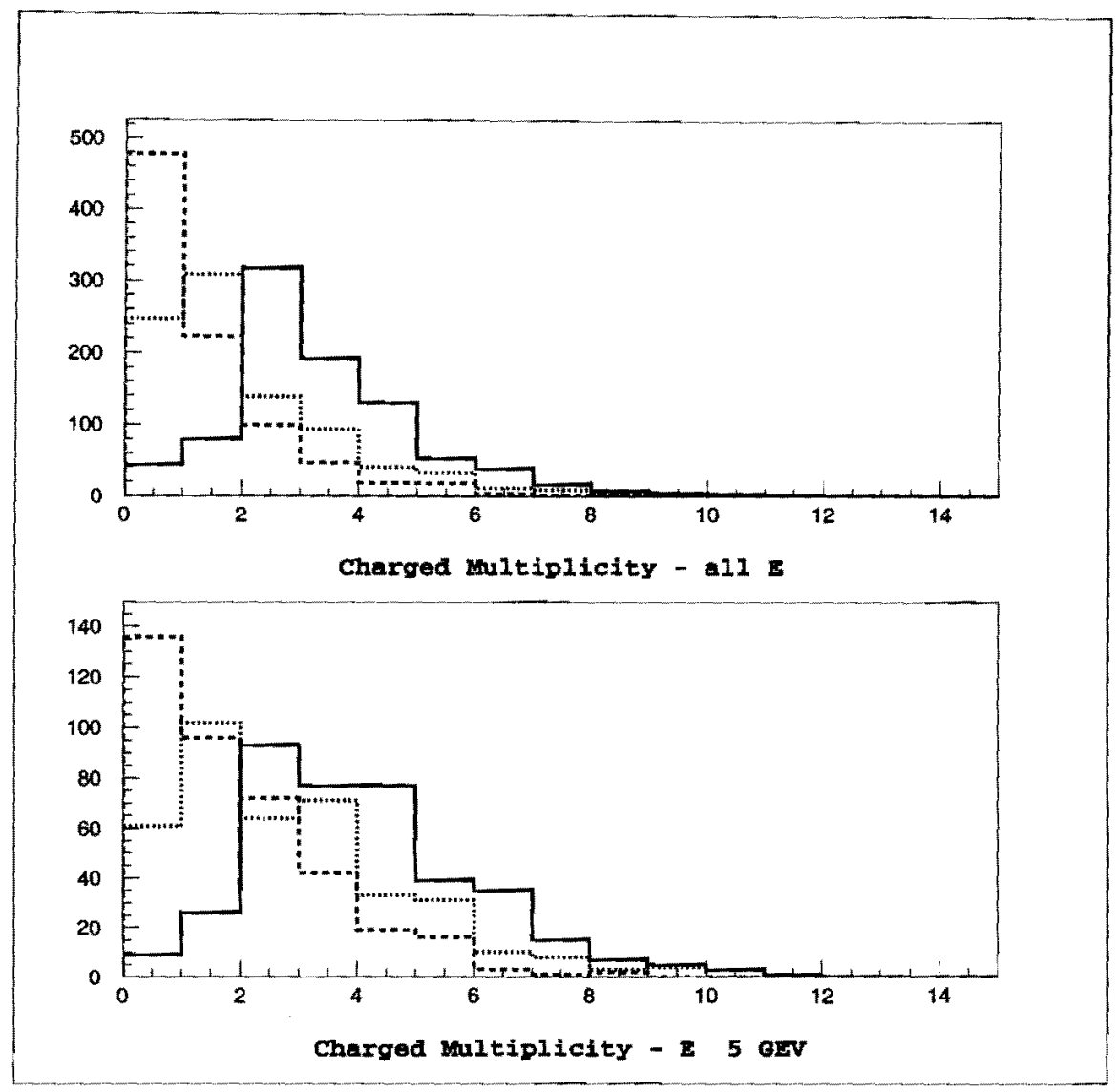

Figure 9: Charged particle multiplicity for interactions $\left(\nu_{\mu} \mathrm{CC}\right.$ events) from the NuMI LE beam. The dotted lines represent the multiplicity distributions for selecting tracks within the angular restrictions of 1000 and $400 \mathrm{mrad}$. 


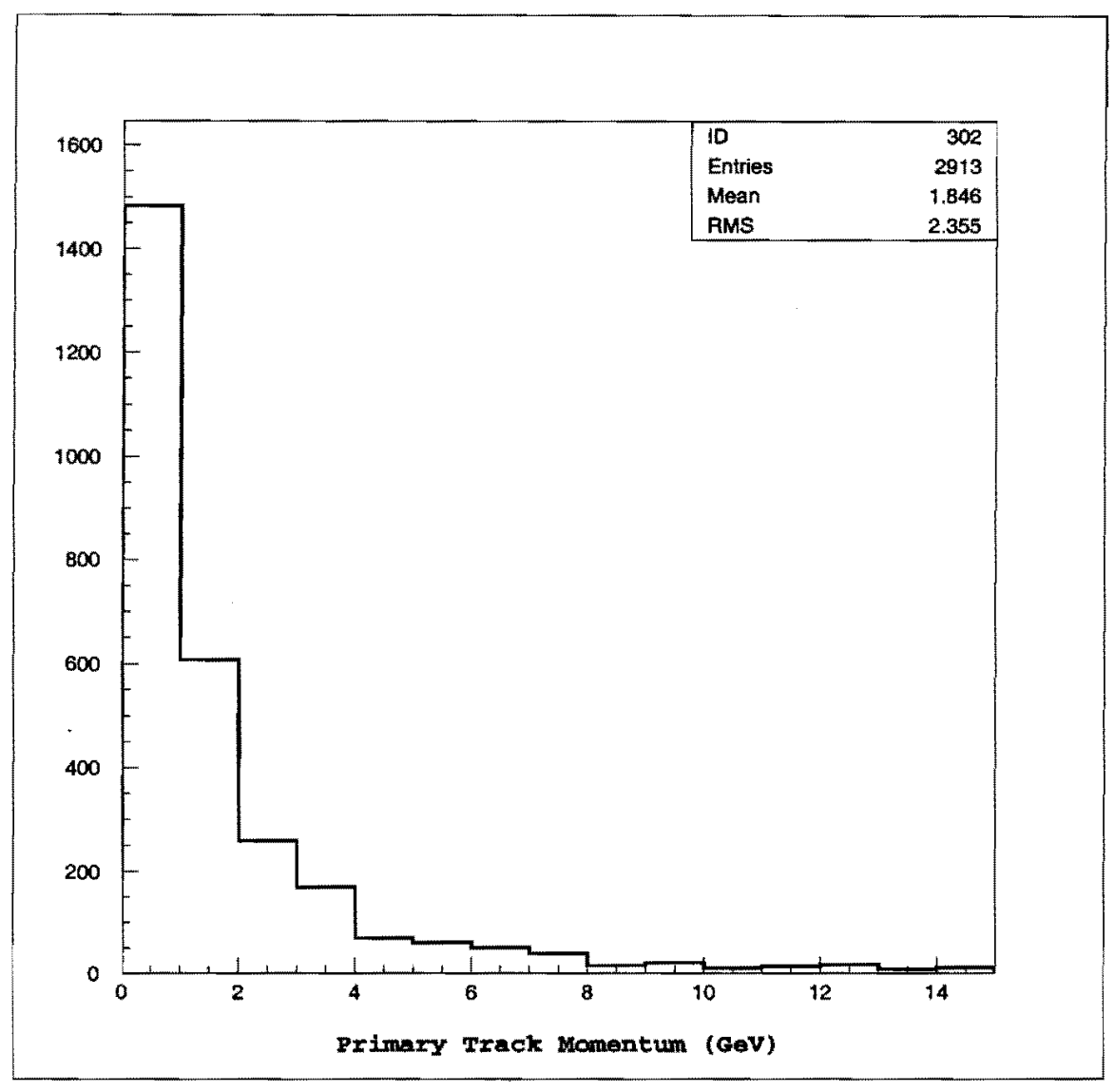

Figure 10: Charged particle momentum for interactions $\left(\nu_{\mu} \mathrm{CC}\right.$ events) from the NuMI LE beam. 


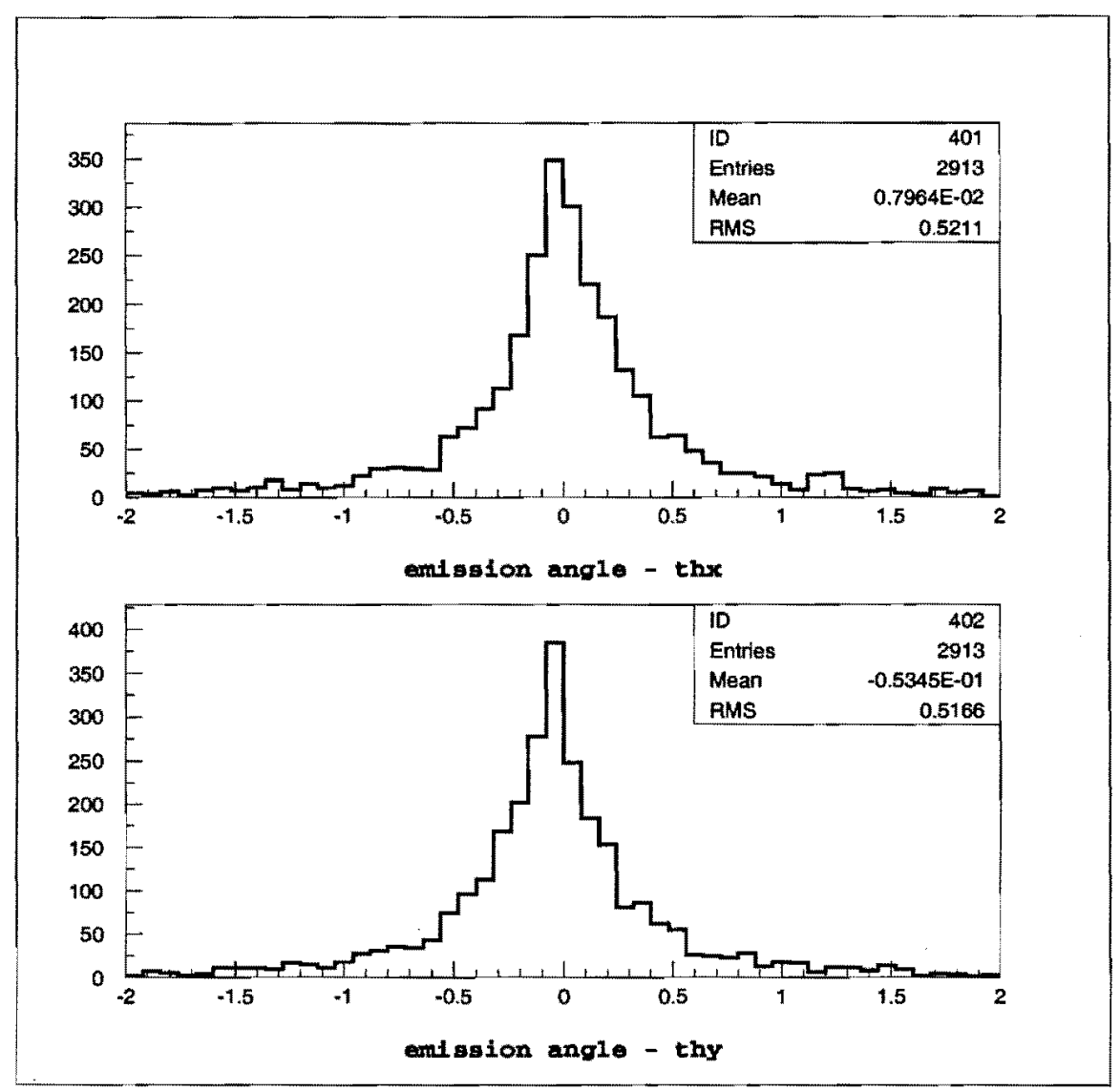

Figure 11: Charged particle emission angles for interactions ( $\nu_{\mu}$ CC events) from the NuMI LE beam. 


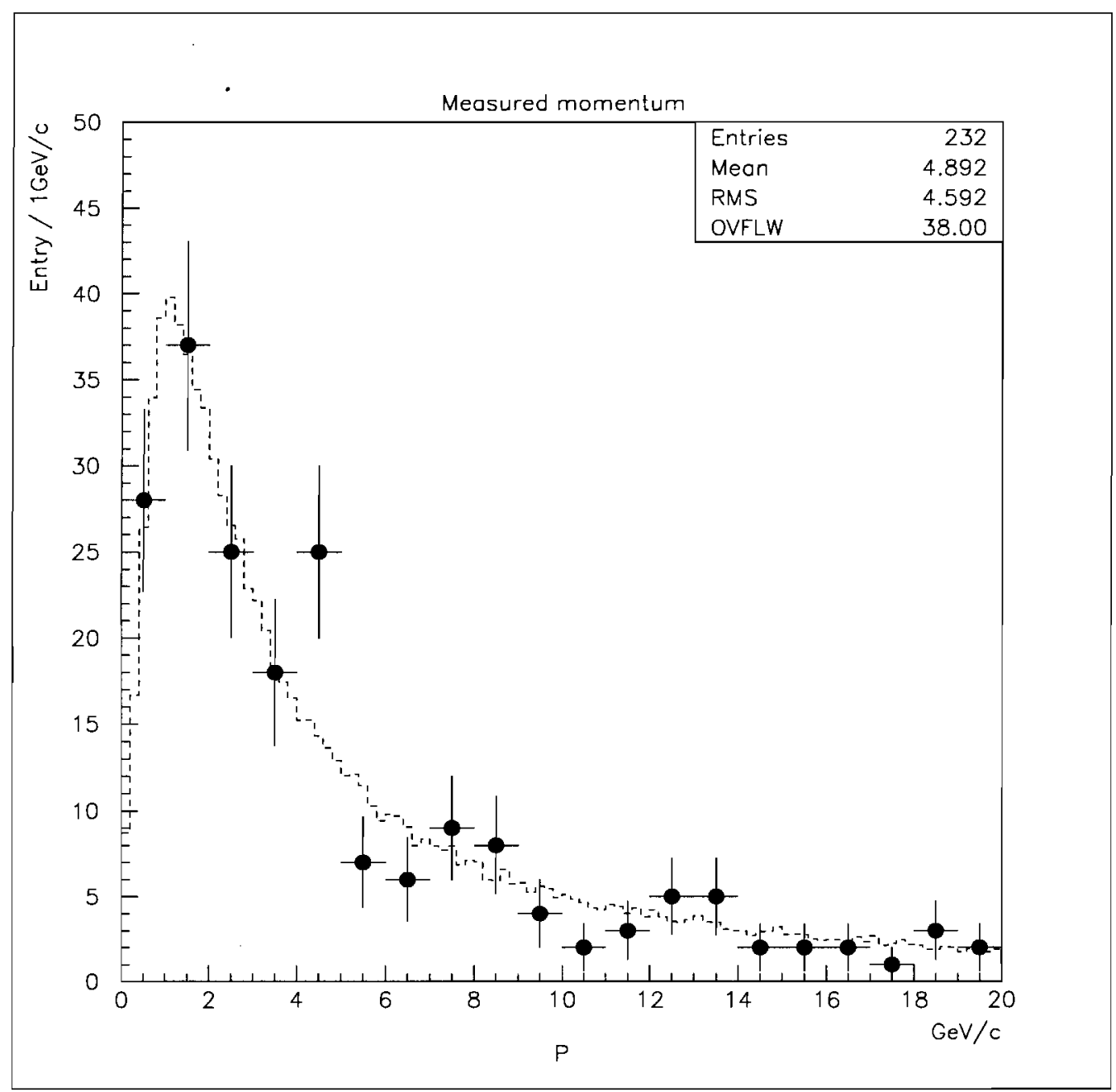

Figure 12: Measured charged track momentum in the DONuT experiment. Plots shows data and dashed line is MC 


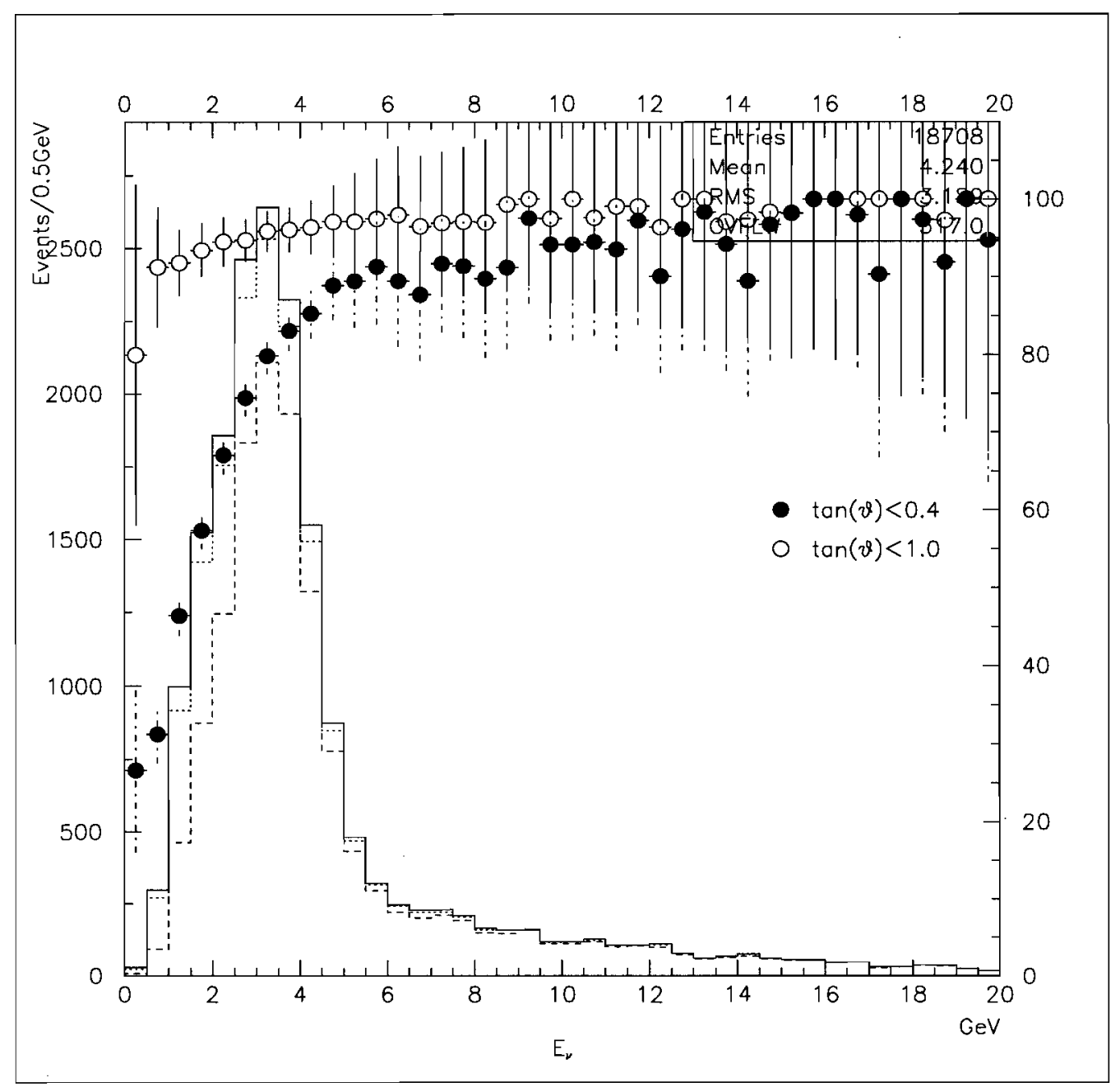

Figure 13: Event location efficiency as a function of neutrino energy. Close and open circle shows location efficiency which using track slope smaller than $\tan (\theta)<0.4$ and $\tan (\theta)<1.0$, respectively 